Revue européenne des sciences sociales

European Journal of Social Sciences

XLIV-134 | 2006

Quel(s) défi(s) pour les sciences sociales à l'heure de la mondialisation?

\title{
La dynamique des échanges de services au fil de la vieillesse
}

Christian Lalive d'Épinay

\section{(2) OpenEdition}

Journals

Édition électronique

URL : http://journals.openedition.org/ress/295

DOI : $10.4000 /$ ress.295

ISSN : 1663-4446

Éditeur

Librairie Droz

Édition imprimée

Date de publication : 1 juin 2006

Pagination : 169-180

ISBN : 9-782-600-01095-5

ISSN : 0048-8046

Référence électronique

Christian Lalive d'Épinay, «La dynamique des échanges de services au fil de la vieillesse », Revue européenne des sciences sociales [En ligne], XLIV-134 | 2006, mis en ligne le 14 octobre 2009, consulté le 03 mai 2019. URL : http://journals.openedition.org/ress/295 ; DOI : 10.4000/ress.295 


\title{
Christian LALIVE D'ÉPINAY
}

\section{LA DYNAMIQUE DES ÉCHANGES DE SERVICES AU FIL DE LA VIEILLESSE}

\author{
Pour Gérald, \\ un grand-père qui s' exerce à l'art du don
}

\section{LIEN FILIAL ET PARADIGME DU DON}

Pour Gérald Berthoud ${ }^{1}$, dans la suite de Marcel Mauss, le don est « une mise en sens» en même temps qu'une «mise en relation»; il «fonctionne en même temps qu'il signifie». Condition du «tenir ensemble d'une société», le don est en même temps l'affirmation d'une appartenance sociale et un exercice de différentiation qui rend manifeste l'individualité du donneur.

Alors que dans ses travaux sur le don, Mauss s'est surtout intéressé au moment du «rendre» et à l'obligation qui lui est associée, Berthoud de son côté veut scruter le premier moment du cycle, «celui du 'donner', à partir du mobile défini par Mauss comme 'caractère volontaire'»: la volonté donatrice. Selon lui, le caractère premier du donner, puisqu'il instaure l'échange, fait que le système d'échange n'est jamais horizontal, symétrique. Il exprime sans doute une volonté de partager avec autrui, donc il manifeste une solidarité, mais dans cette «action généreuse » le donneur «affirme sa supériorité» de créateur.

Tout acte de donner renvoie au don initial, le don de la vie, donc aussi au don originel, celui de la création de la vie. Berthoud signale que les sociétés communautaires s'accordent à penser que «dans les premiers temps du monde humain, tout a été donné ». Les grandes religions monothéistes n'affirment rien d'autre. En va-t-il autrement dans le nouveau «grand récit de la vie $»^{2}$ auquel adhèrent, nolens volens, les agnostiques de notre temps? Les premiers temps, les sept jours de la création biblique, s'y dilatent en une histoire couvrant des milliards d'années, avec du désordre et du chaos, des accélérations, des paliers, des bifurcations, mais le résultat n'est-il pas le même? Pour que la vie puis l'homme émergent, il fallait que «tout soit donné !» Le «Cantique du Soleil» de St François chante le don de

Cette introduction est inspirée par plusieurs écrits de G. Berthoud, mais plus particulièrement par sa présentation d'un état de la théorie du don dans: «Penser l'universalité du don: à quelles conditions?», Revue du MAUSS, n² 23, 2004, p. 353-376.

2 Michel Serres: Hominescence, Paris, Le Pommier, 2001. Si nous rejoignons l'analyse proposée par cet auteur de la formation de ce «nouveau grand discours », nous ne le suivons pas quand il s'en fait l'apologiste. 
la vie en louange à son créateur. Aujourd'hui, pour beaucoup, il n'y a ni créateur ni intelligent designer, si ce n'est la vie elle-même, une vie qui n'est plus si mystérieuse puisque biologistes et paléontologues en retracent l'histoire, mais qui ne cesse pourtant de stupéfaire tant il a fallu de circonstances et de convergences pour canaliser ce processus, unique à ce jour et dans l'état des connaissances! Dès lors, de multiples hymnes à la vie s'élèvent, qui ne s'adressent plus à son supposé créateur, mais à la vie elle-même ! ${ }^{3}$ Berthoud rappelle que beaucoup de sociétés communautaires expriment leur relation au monde au moyen de métaphores empruntées à la parenté. Certains se veulent les «enfants de la forêt», source de tous les biens, lieu de tous les dons. D'autres ont pour créateur «Père-soleil», celui qui donne sans jamais recevoir. Mais voilà qui n'est pas limité à ces sociétés: Déméter aux multiples seins ne symbolise-t-elle pas pour les Grecs la fertilité? Les écologistes contemporains n'invoquent-ils pas Gaia, la terre-mère?

\section{LE DON DE LA VIE, À L'ORIGINE DE LA FAMILLE}

Si la parenté a offert aux hommes les images et les mots pour désigner et interpréter leur rapport au monde et aux origines, ne convient-il pas de retourner la relation pour s'interroger sur ce que les mythes de la création disent de la parenté? Relevons d'abord qu'ils s'attachent à un élément bien spécifique du système de parenté, celui qui unit les géniteurs (père et mère) à ceux qu'ils engendrent (les enfants). Ce don de la vie fonde la famille et la reproduit. A une condition cependant, ce don doit être reconnu comme tel. Sans la reconnaissance - au sens ici de prendre acte de son action créatrice et d'en accepter la proclamation publique -, la relation parentale n'existe pas. Le don de la vie, dès l'instant où il est reconnu, produit une relation. Cette relation instaure une communauté : la famille, en même temps qu'elle produit de l'identité individuelle, donc différentielle, par une distribution de rôles: père et mère d'un côté, fils ou fille de l'autre. Parce qu'elle unit deux générations, cette relation est verticale et radicalement asymétrique. A son origine, la relation parent-enfant ${ }^{4}$ associe un homme et une femme adultes et un petit d'homme qui n'est à ce stade qu'un potentiel d'être radicalement hétéronome. Pour celui qui le reçoit, le don de la vie se traduit, dans un premier temps tout au moins, par un état de dépendance totale envers ses donneurs. La réalité de la naissance d'une vie humaine se démarque radicalement de la fiction mythique qui elle fait surgir un être humain à l'état adulte. Dans le mythe, la distance séparant la créature du principe créateur est sans doute affirmée, il n'empêche que la créature naît à la vie adulte et autonome (une autonomie signalée par le mythe de l'arbre du bien et du mal, par ex.): tout lui est donné. Dans la genèse du lien

Citons le splendide «Gracias a la vida...» de Violetta Para, à qui le renouveau de la musique et de la chanson andine doit tant. Ce poème a valeur de testament, Violetta Para s'étant donné la mort peut après l'avoir écrit. Signe que le don de la vie peut être chanté alors même qu'on a choisi de la quitter.

4 Je parlerai de relation filiale, m'écartant quelque peu de la définition conventionnelle pour désigner ce qui lie les géniteurs à leurs enfants, et ces derniers à leurs parents. 
parental, l'asymétrie entre donneur et receveur, la toute puissance du premier, la dépendance radicale du second, sont bien plus abyssales encore que dans les mythes de la genèse: tout est loin d'être donné au nouveau-né. Mais en même temps s'observe un paradoxe: du fait même de cette dépendance radicale, le bénéficiaire du don n'est pas (pas encore?) l'obligé; du fait de son pouvoir sans limite, le donneur n'est pas (pas encore?) l'obligeant. Bien au contraire, l'asymétrie radicale du don de la vie, oblige le donneur plutôt que le receveur: les parents « reçoivent» l'enfant qu'ils ont engendré. Il est d'abord un don pour eux, et les voilà, le plus souvent enchantés mais toujours obligés par ce don.

$\mathrm{Si}$, comme le signale Berthoud, les récits mythiques ont largement emprunté à la parenté pour raconter et signifier le don initial de la vie, n'est-ce pas tout simplement parce que, dans l'expérience humaine, rien ne symbolise mieux la fertilité que la grossesse de la femme et le don de la vie que la naissance de l'enfant? Dès lors, dans quelle mesure le paradigme du don s'offre-t-il en cadre heuristique pertinent pour l'interprétation du système dynamique des échanges entre parents et enfants, tant dans leur dimension symbolique que dans celle des pratiques?

\section{DE LA DYNAMIQUE DU LIEN FILIAL}

Quoi de plus dynamique, de plus sujet à transformation multiple que la relation entre les géniteurs et leurs enfants? L'invariant est le fait de la filiation, qui perdure quelle que soit la manière dont évolue la relation. Mais le contenu des termes ainsi reliés et de la relation elle-même ne cesse de se transformer. A chaque étape de la vie, qui est donneur, qui est receveur, et qu'est-ce qui se donne ou s'échange? Dans un premier temps, au fur et à mesure que s'accomplit la mutation de l'enfant en adulte, la relation perd de sa verticalité et gagne en symétrie. Vient le temps délicat de l'adolescence, avec pour les parents la perte de leur toute puissance, l'apprentissage de la prise de distance, d'une distance qui n'est pas absence, mais discrétion, attention, disponibilité. Se met en place, avec le départ des enfants du foyer familial, une relation d'adulte à adulte, entre adultes de deux générations. Voilà qui est particulièrement vrai dans le cadre des sociétés occidentales et de la culture post-moderne, où la valeur d'autonomie individuelle se présente comme la finalité de l'activité pédagogique, celle de la famille comme celle de l'institution scolaire. Mais le modèle patriarcal n'a pas totalement disparu; sans même parler de sa présence dans la culture de certaines populations migrantes, rappelons que notre culture bourgeoise le véhiculait jusqu'à peu. On sait aussi ce qu'il advient des enfants qui n'ont pas su résister à des parents abusifs, et le qualificatif désigne l'excès dans l'exercice de l'autorité, l'abus de séduction envers ses enfants, et le fait de n'avoir ni su ni voulu se départir du rôle de patriarche (ou matriarche) au fil de la maturation de ses enfants. La littérature sur les parents abusifs s'intéresse avant tout à l'enfant, mais quelques travaux suggèrent que cette attitude n'est pas sans implication sur la vieillesse des parents abusifs. Par exemple, la monographie d'une pension a mis en lumière que les vieillards délaissés par leurs enfants se comportèrent dans une phase de vie antérieure en parents abusifs dont les enfants n'ont pu conquérir leur autonomie qu'au 
prix d'une rupture, une rupture jamais admise, jamais comprise par le géniteur qui se refuse de reconsidérer son rôle et qui ainsi rend la relation impossible 5 .

La transition de l'adolescence est lourde d'enjeux, car de fait s'y joue tout l'avenir de la relation; elle est tragique, puisque sur le plan symbolique, elle implique pour les parents la perte de l'enfant, et pour les enfants, le meurtre des parents; elle est aujourd'hui d'autant plus périlleuse que si l'on voit ce qui se déconstruit, ce qui se reconstruit ne peut plus s'appuyer sur des modèles culturels prescrits. Tout se passe, à ce stade, comme si se reconstruisait une relation incertaine, fluide, dans laquelle le don et ses corrélats (donneur, receveur, passeur, échange, reconnaissance, obligation) sont mis entre parenthèses, présents mais de manière latente. Ils ne structurent plus la routine relationnelle; ils sont comme suspendus, désactivés mais présents, et se voient réactivés sous l'impact de l'événement: le mariage, la naissance d'un (petit-) enfant, Noël, mais aussi la maladie, la crise, le chômage, le divorce...

\section{LA RETRAITE, ÂGE DU DON?}

La retraite des parents va marquer une nouvelle transition. Dans l'immédiat après-guerre, lors de sa création, cette institution avait pour but de soulager les personnes vieillissantes, en leur épargnant la misère et la déchéance qu'engendrait la nécessité de continuer à travailler pour vivre quand bien même le corps ou l'esprit n'en ont plus la capacité. Mais depuis lors, l'espérance de vie ne cessant d'augmenter et l'état de santé des personnes vieillissantes de s'améliorer, la retraite ouvre sur une situation radicalement nouvelle et surprenante. Voici le retraité néophyte, «âgé mais pas vieux», doté d'un revenu le plus souvent (dans notre pays) confortable, donc libéré de l'obligation de travailler pour vivre. Pour la première fois de sa vie, les clés de son temps lui sont remises; le voici placé dans un état de disponibilité générale, qui l'invite à réorganiser sa vie selon ses choix, donc selon un «caractère volontaire» pour reprendre cette catégorie de Mauss. Qui plus est, en le retirant du monde économique de la production, elle le libère du critère de rentabilité à l'aune duquel il était jusqu'ici jugé: ses choix ont maintenant tout le loisir de faire la plus large place à la gratuité, au don. Enfin, en l'écartant de cette pièce centrale de l'espace public qu'est l'entreprise ${ }^{6}$, elle l'oriente naturellement vers l'espace privé, les amis, la famille.

La retraite instaure ainsi, en champ du possible, l'âge du don. Dans quelle mesure le retraité fait-il de ce potentiel une réalité, en particulier dans le cadre de sa famille?

Cette question est d'autant plus pertinente qu'une autre ligne de force culturelle pourrait faire obstacle à une telle réorientation de la vie. Il n'est guère discu-

\footnotetext{
5 Cristina Ferreira, Placement et familles: le poids de l' histoire familiale. Mémoire de maîtrise, Département de Sociologie, Université de Genève, 1997.

- Pièce centrale mais non pièce unique: dans le système économique le retraité n'est plus producteur, mais son statut de consommateur n'en prend que plus d'importance; par ailleurs, il n'en demeure pas moins citoyen, et si la tendance est de traiter les retraités en citoyens de seconde zone, ils disposent de toutes les cartes pour façonner et affirmer leur rôle civique.
} 
table que la culture contemporaine sécrète un individualisme qui fait du «je » et du sentiment subjectif d'épanouissement des valeurs cardinales ${ }^{7}$. La retraite ne se présente-t-elle pas comme l'occasion par excellence - comme l'occasion ultime de s'adonner à cette quête d'épanouissement que jusqu'ici tant de contraintes - le travail, mais aussi ... la famille et les enfants! - entravaient. L'économie, qui n'a pas tardé à prendre acte du potentiel consommatoire d'une population de rentiers, développe une très large offre de "produits» qui se prétendent sources d'épanouissement, des voyages dans les îles paradisiaques à la chirurgie esthétique, en passant par la gamme infinie des poudres à perlimpinpin de l'antiaging. Qu'en est-il dans les faits? ${ }^{9}$

Dans le cadre de deux études de la population âgée menées l'une en 1979 et l'autre 15 ans plus tard, en 1994, sur la base d'un inventaire des activités des retraités, nous avons dégagé cinq styles de vie. Le premier est caractérisé par un très faible niveau d'activité dans l'ensemble des domaines; le second - repli désigne un style de vie essentiellement cantonné dans l'espace domiciliaire. Le suivant - participation - est centré sur la participation aux associations volontaires, et sur les activités bénévoles et de services. Le quatrième - participation et loisirs - combine les engagements et les services avec des loisirs d'épanouissement, tels les voyages, les activités physiques et récréatives. Le dernier est un mode d'organisation de la vie autour des loisirs visant à l'épanouissement personnel. Il exprimerait donc cette quête individualiste du Graal contemporain.

Tableau 1: Les styles de vie dans la population de 65 à 79 ans (en \% d'adeptes): 1979 et 1994.

\begin{tabular}{|l|c|c|c|}
\hline Style de vie & 1979 & 1994 & Différence (1994/1979) \\
\hline Peu actifs & 23 & 15 & -8 \\
Repli & 21 & 16 & -5 \\
Participation & 19 & 18 & -1 \\
Loisirs et participation & 16 & 25 & +9 \\
Loisirs & 21 & 26 & +5 \\
\hline Total & 100 & 100 & \\
\hline
\end{tabular}

Le tableau 1 met en lumière la désaffectation marquée des deux styles de vie les plus passifs et repliés, ce qui résulte pour une bonne part du meilleur état de santé de la génération la plus récente. De manière complémentaire, ce sont les

cf. Christian Lalive d'Epinay, «Significations et valeurs du travail, de la société industrielle à nos jours », in: M. de Coster et Fr. Pichault (sous la dir. de), Traité de sociologie du travail, Bruxelles, de Boeck, 1994, p. 55-82; Alain Ehrenberg: La fatigue d'être soi, Paris, Odile Jacob, 1998.

\& Car depuis une dizaine d'années on est passé des doctrines du successful aging à l'idéologie du antiaging, de la volonté de relever le défi de la vieillesse à la prétention de la nier. Voir à ce propos l'article de Cornelia Hummel: «La vieillesse, un âge obsolète?», in: S. Cavalli et J.-P. Fragnière (éds), L'avenir. Attentes, projets, (dés)illusions, ouvertures, Lausanne, Réalités sociales, 2003.

9 Je m'appuie ici sur un ensemble de recherches menées sous ma direction dans le cadre du Centre Interfacultaire de Gérontologie (CIG) de l'Université de Genève. Voir entre autres: Christian Lalive d'Epinay, Jean-François Bickel, Carole Maystre et Nathalie Vollenwyder, Vieillesses au fil du temps: 1979-1994, une révolution tranquille, Lausanne, Réalités sociales, 2000; Christian Lalive d'Epinay et Dario Spini (sous la dir. de), Les Années fragiles: La vie au-delà des 80 ans, Québec, Presses universitaires de Laval, 2006 (sous presse). 
styles de vie «loisirs » et avant tout «loisirs et participation» qui gagnent le plus d'adeptes; la quête de l'épanouissement personnel est propre à un quart des personnes du 3ème âge, mais un autre quart entend combiner les engagements et le don de soi avec la jouissance de plaisirs typiques de la modernité. Comme par ailleurs, un retraité sur cinq environ fait de la participation sociale et du service à autrui l'axe principal de son organisation de la vie, c'est au total une petite moitié $(43 \%)$ des personnes du troisième âge qui ménagent une place nucléaire à l'engagement, au volontariat et au service d'autrui.

La part accordée à l'activité donatrice est ainsi loin d'être négligeable dans l'univers de vie des retraités. Mais de celle-ci qu'est-ce qui revient à la famille? Toutes les études convergent à montrer que celle-ci constitue le lieu principal de l'exercice de cette forme d'activité ${ }^{10}$.

En ce qui concerne les dons matériels, des études françaises et américaines ont montré que les flux circulaient avant tout selon une ligne descendante, des générations les plus anciennes aux plus récentes ${ }^{11}$. Mais intéressons-nous avant tout aux dons prenant la forme de services rendus, puisque c'est avant tout du temps que le retraité se voit offrir presque à discrétion. Le tableau 2 (voir page suivante) indique la position occupée par les personnes âgées (donneur, receveur, échangeur - la personne rend des services et en reçoit -, et enfin les personnes non insérées dans un tel système). Dans la première partie du tableau (col. 1), on considère un échantillon de personnes âgées de 65 à 79 ans et interviewées en 1994; dans le reste du tableau (col. 2), nous donnons d'abord la distribution des personnes âgées de 80 à 84 ans en 1994 selon leur position dans le système d'échange (col. 2.1), personnes que nous avons retrouvées cinq ans plus tard, en 1999 (col. 2.2), ce qui permet d'observer les changements de positions qui se sont opérés dans ce laps de temps.

Observons d'abord la première partie du tableau (col. 1). Parmi les personnes du 3ème âge (65-79 ans), les receveurs sont une petite minorité, et l'analyse a montré qu'il s'agit avant tout de personnes fortement atteintes dans leur santé. Par ailleurs, près d'une sur cinq, n'intègre pas le réseau d'échange avec la famille; un examen de détail montre qu'il s'agit dans la plupart des cas de personnes sans famille proche ${ }^{12}$. Une moitié occupe une position de donneur; au total, dans le 3ème âge, presque toutes les personnes insérées dans un réseau familial d'échange sont prestataires de services.

${ }^{10}$ Principal mais non exclusif: les engagements citoyens des retraités sont loin d'être négligeables, les relations d'amitiés sont nourries d'attentions réciproques, le voisinage dans un immeuble suscite plus souvent qu'on ne le pense des échanges et des formes d'entraide. C'est à cette question que J.-Fr. Bickel a consacré sa thèse: Citoyenneté et participation au temps de la retraite, thèse doctorale, Département de Sociologie, Université de Genève, 2003.

${ }^{11}$ Claudine Attias-Donfut, «Rapports de générations. Transferts intrafamiliaux et dynamique macro sociale», Revue Française de Sociologie, vol. 41/4, p. 643-684.

12 Signalons que la famille que dessine le système des échanges se compose des ascendants, quand il en est de survivants, de la fratrie, et des descendants. En cas d'absence de descendants, les neveux et nièces sont introduits dans le système. Sauf de rares exceptions, la famille proche n'a pas d'autres composantes. Du point de vue de la théorie du don, observons que la famille proche comprend ceux dont on a reçu le don de la vie, ceux qui ont reçu ce même don des mêmes personnes, et ceux à qui à son tour on a donné la vie (ou, dans le cas des neveux, à leurs substituts). 
Tableau 2: Position des personnes de 65 à 69 ans dans le système d'échange de services au sein de la famille (en \%)

\begin{tabular}{|c|c|c|c|}
\hline \multirow[t]{2}{*}{ Position } & \multirow{2}{*}{$\begin{array}{c}(1) \\
\text { Personnes } \\
\text { de } 65 \text { à } 79 \text { ans } \\
(1994)\end{array}$} & \multicolumn{2}{|c|}{$\begin{array}{c}\text { (2) } \\
\text { Cohorte d'octogénaires }\end{array}$} \\
\hline & & $\begin{array}{c}(2.1) \\
\mathrm{l}^{\mathrm{er}} \text { entretien } \\
(80-84 \text { ans })\end{array}$ & $\begin{array}{c}(2.2) \\
5 \text { ans plus tard } \\
(85-89 \text { ans })\end{array}$ \\
\hline $\begin{array}{l}\text { Donneurs } \\
\text { Echangeurs } \\
\text { Receveurs } \\
\text { Hors système }\end{array}$ & $\begin{array}{r}47 \\
30 \\
5 \\
18\end{array}$ & $\begin{array}{l}20 \\
30 \\
20 \\
30\end{array}$ & $\begin{array}{r}8 \\
24 \\
38 \\
30\end{array}$ \\
\hline & $100 \%$ & $100 \%$ & $100 \%$ \\
\hline
\end{tabular}

Qui sont les destinataires principaux des services offerts par les retraités? Dans la mesure où ceux-ci, longévité aidant, ont encore un parent en vie mais qui par la force des choses a atteint un âge plus que respectable, ce dernier - dans la règle une femme - est l'objet d'une attention particulière. La relation qui unit l'enfant âgé à son parent survivant est-elle l'occasion du contre-don, le don en retour du don initial? On y reviendra dans le prochain paragraphe, mais retenons ici que l'ascendant survivant de la famille apparaît comme le destinataire obligé du don de disponibilité, donc de temps.

Sur l'ensemble cependant, le premier destinataire (le plus fréquent et le récipiendaire du plus grand nombre de services) est l'un ou (et) l'autre de ses propres enfants, tout particulièrement quand cet enfant a fondé sa propre famille, donc installé ses parents dans le statut de grands-parents. Il est vrai qu'aujourd'hui tout incite à cette forme de don: l'activité professionnelle des deux jeunes parents, et aussi les divorces et les familles pudiquement baptisées monoparentales. Pourtant, pour les retraités, s'agit-il seulement de «rendre service»?

Les récits de retraités que nous avons réunis dans le cadre d'entretiens semidirectifs font apparaître la multivalence propre à l'acte de rendre service. Les retraités ne manquent pas de souligner l'importance objective des services qu'ils rendent («Sans nous, elle [notre fille] ne pourrait avoir le poste qu'elle occupe»), les sacrifices consentis («Un ami m'a invité à partir en voyage avec lui, mais j'avais promis à mes enfants...»), le fait que l'activité ne s'accomplit pas sans effort ( «Ils - les petits-enfants - ont une telle vie, après, quand ils sont partis, moi je suis épuisée»); mais bien vite ils confessent leur bonheur à passer du temps avec leurs petits-enfants, à les voir grandir, à leur enseigner des savoirs-faire; au point que le service rendu (et sa valeur utilitaire) apparaît comme un heureux prétexte à cultiver une relation qui pour eux est vitale, tant il est vrai que la création d'une relation grand-parentale réactive le lien filial. Les activités grandparentales rendent manifeste que dans le don, le social l'emporte sur l'économique, et l'affectif sur le rationnel: «quand elle (ma fille) me demande de faire quelque chose pour elle, parce que dans la journée, pour elle c'est difficile, alors je le fais et c'est comme si j'étais avec elle!», confie un veuf.

Ainsi, après des années où la part du lien filial animée par le don paraissait comme suspendue, ne s'actualisant plus qu'à l'occasion de rituels ou d'événements, la retraite, particulièrement lorsqu'elle se conjugue avec la grand-parenta- 
lité, crée pour la génération aînée l'occasion de reprendre sa position de donneur et, ce faisant, de se réinsérer dans un système familial dont, dans un premier temps - celui du mariage des enfants - elle s'était trouvée sinon exclue du moins marginalisée. Mais ce nouveau statut de donneur diffère largement du statut initial associé à la condition de géniteurs: celui-ci relevait de l'obligation associée à une «mission» confiée aux parents et codifiée dans la législation; celui-là est maintenant totalement volontaire, la reconnaissance qui en découle de la part des enfants réside moins dans l'émission d'une créance (reconnaissance de dette) par les enfants pour «services rendus » des parents, que dans la réintégration des grandsparents dans un système d'échange élargi puisque tri-générationnel. Un grandparent ne reste-t-il pas un être virtuel tant qu'il ne prend pas place dans le système des échanges?

\section{LA VIEILLESSE AVANCÉE: NE PLUS POUVOIR DONNER, ACCEPTER DE RECEVOIR}

Mais que se passe-t-il quand la vieillesse se fait pesante? Quand la fragilisation liée au grand âge porte atteinte à la capacité de donner du parent?

Retournons au tableau 2 pour observer ce qui se passe au sein d'une cohorte d'octogénaires ${ }^{13}$. La comparaison des deux premières colonnes montre qu'alors que les trois-quarts de la population du $3^{\text {eme }}$ âge occupent des positions de donneurs ou d'échangeurs, ce n'est le cas que de 50\% des personnes de 80 à 84 ans. Mais qu'est-ce qui paraît le plus surprenant: ce déclin d'un rôle actif au fil de l'âge, ou au contraire qu'un octogénaire sur deux soit à même de préserver son aptitude à donner?

Cela dit, la comparaison des colonnes 2.1 et 2.2, qui montre l'impact du vieillissement sur la structure du système d'échange, met au jour un des enjeux névralgiques de la neuvième décennie de la vie; dans le laps de cinq ans, les grands-parents «soleil» (qui donnent sans recevoir, pour reprendre la métaphore) ont presque disparu et en revanche la proportion de ceux qui maintenant reçoivent sans plus pouvoir donner a passé de 20 à 38\%. Si l'on ne tient pas compte des «hors système» (cf. note 13), presque tous bénéficient de services offerts par des membres de leur famille: la fragilisation affecte l'aptitude à donner, mais stimule le don dans l'entourage familial.

Comme l'attestent les témoignages, si donner devient difficile, il n'en demeure pas moins un enjeu, et si recevoir devient nécessaire, encore faut-il pouvoir l'accepter!

\footnotetext{
${ }^{13}$ Que cette cohorte comprenne $30 \%$ de personnes «hors système» renvoie indirectement à sa spécificité démographique. Alors que leurs cadets de 65 à 79 ans font partie des «papy boomer», eux se sont montrés peu prolifiques, particulièrement dans le contexte urbain. Par ailleurs, ils n'ont plus d'ascendants à entourer.
} 


\section{Donner, tant qu'on peut!}

«Je suis solide. Physiquement je suis solide, j'ai 85 ans, j'ai du rhumatisme dans le dos, mais j'ai pas de maladie. Alors je peux quand même servir! (...) Dès que je peux aider, j'aide; cela c'est certain. Et on trouve toujours!»

«Maintenant je tricote pour le petit bébé né il y a un mois.

- L'arrière-petit-fils?

- Oui, voilà. Et puis j'aimais bien tricoter, mais je n'arrive plus tellement. La vue baisse et puis les mains ça tremblote...»

\section{Les dons encore possibles}

A propos de ses petits enfants: «on s'entend bien avec les jeunes: on a plus de temps pour les écouter quand on est vieux.»

«(J'ai toujours) des bonbons pour les enfants. Bien qu'on dise qu'il ne faut pas leur donner des bonbons... mais enfin, ils sont trop déçus si je n'ai pas des bonbons (rires)!»

\section{Ne plus pouvoir...}

Les récits présentent la longue litanie de ce qu'on faisait, mais qu'on ne peut plus faire - «maintenant, ça, c'est fini !». Difficile renoncement, quand on ne peut plus s'occuper de ses petits- ou arrière-petits-enfants, parce qu'on n'est plus à même d'en assurer la sécurité et que leur vivacité n'est pas sans danger pour le vieillard.

\section{Du sentiment d'inutilité...}

«On se sent maintenant un peu inutile, parce qu'on s'est toujours occupé de quelque chose ou de quelqu'un. Maintenant on ne s'occupe plus que de soi-même, alors c'est ça qui pèse. On se sent bien inutile.

- Vous vous sentez inutile?

- Tout à fait inutile, je ne peux plus rien faire pour les autres. Tout ce que je fais, c'est pour moi. Et les autres font pour moi, bien sûr, mais ... non, je ne peux plus rien faire, plus rien faire.»

\section{... à celui d'être un souci pour autrui}

«On devient trop vieux (...) On est quand même une charge, une personne âgée; c'est quand même un souci (...) C'est comme aujourd'hui, j'ai eu un petit malaise là. Je ne sais pas ce qui m'a pris. Voyez mon petit-fils m'a tout de suite appelée pour savoir comment ça va. Il se fait du souci, c'est ça qui est embêtant.»

\section{La permutation des rôles}

«Maintenant, j'ai un neveu, je ne sais pas quand il vient, mais c'est lui qui fait tout, mes services, les courses (...) Des fois il me dit: 'Tu es libre? Tu viens avec moi', et puis je vais avec lui, je fais un tour (...) Parce que lui, il fait tout, il est comme le père à moi.»

«- Est-ce que vous avez un conseil à donner aux personnes qui, comme vous, deviennent très âgées? 
- Se faire aider assez tôt. Oui, accepter de l'aide assez tôt. Je vois une femme, sa fille voulait la prendre chez elle, mais elle n'acceptait pas, elle voulait rester chez elle (...) Elle avait une minerve et les mains toutes recroquevillées. Mais elle ne voulait pas partir de chez elle. Maintenant elle est dans un home, la fille ne pouvait plus s'en occuper assez.

Accepter de se faire aider. Ce n'est pas une honte, c'est une nécessité. Et puis penser que c'est une chose naturelle, la vie s'écoule comme ça, comme un torrent ! Des fois il y a des pierres, des fois y'en a point! (rires).»

Ces extraits montrent à quel point il en coûte de devoir renoncer à être un donneur. Le: «maintenant je ne peux plus » a souvent été jeté à la tête de l'enquêteur comme un cri d'impuissance confessée, mais mal assumée. «Je ne peux plus » laisse poindre un «je n' en peux plus », un «je vis encore mais je ne suis déjà plus des vivants».

De multiples stratégies sont mises en place pour préserver en la réaménageant l'aptitude à donner. Nous venons d'en voir deux, l'une, très riche, consiste dans la disponibilité de l'écoute. L'autre minimaliste, dans l'offre de bonbons. Mais qui n'a pas en tête ces visites à des vieillards qui, à un moment ou à un autre, tenaient à offrir quelque chose, une douceur ou encore un peu d'argent? «Tiens, c'est pour toi !» Continuer à donner pour continuer à exister.

Si ne plus être à même de donner est douloureux, alors on comprend que devoir être aidé, sans plus pouvoir donner le soit plus encore pour beaucoup. Mauss enseigne que dans le don, celui qui donne manifeste une supériorité. $\mathrm{Ne}$ plus pouvoir donner prive de cette supériorité, devoir recevoir atteste une infériorité. Le comportement tyrannique, jamais satisfait, jamais reconnaissant de certains vieillards dépendants ne doit-il pas être compris à travers le prisme de la frustration engendrée par le constat de leur impuissance?

Pour ne pas se sentir humilié par l'obligation de recevoir, il faut, semble-t-il, être à même de lui donner du sens, de produire une interprétation qui légitime le statut de receveur. Et certains vieillards se découvrent les Monsieur Jourdain d'une théorie de l'échange et du don dans le cours du cycle de vie: «Maintenant, c'est mon tour!» «Toute ma vie, j'ai donné, à mes enfants, à mon mari, aux proches. A moi maintenant de recevoir.» Le contre-don a été différé jusqu'à ce jour, mais maintenant, il est juste qu'il se manifeste. «C'est une chose naturelle, la vie s'écoule comme ça.» Quand la vieillesse s'associe à la maladie et à l'incapacité, alors l'heure est venue du contre-don. Reçue comme tel, l'aide apportée est alors acceptable.

\section{CONCLUSION}

Dans cet essai, j'ai cherché à traiter la théorie maussienne du don comme cadre heuristique pour interpréter le lien filial. Si mon objectif était de considérer avant tout sa dynamique au cours des dernières étapes de vie des géniteurs, il convenait de saisir ce lien dans sa genèse et d'en considérer le développement.

L'analyse proposée suggère que les donneurs originels, les pères et les mères, ne se départissent pas aisément de cette position dans le système d'échanges. Sans doute acceptent-ils, encore que ce ne soit pas toujours le cas, que l'enfant en 
devenant adulte s'émancipe du lien de dépendance que structure le don, puis de se voir en quelque sorte excentrés du système familial lorsqu'à leur tour, leurs propres enfants donnent la vie. S'installe une période où l'échange, horizontal et bi-directionnel, s'exerce de manière intermittente, soit lors de rituels festifs, soit lorsque l'événement en offre l'opportunité ou en crée le besoin. Mais voici que la retraite, en libérant le temps des parents vieillissants, offre à ceux-ci l'occasion de se réaménager un rôle légitime et apprécié de donneur, et de réintégrer pleinement le circuit des échanges familiaux qui maintenant circulent au sein d'un système tri-générationnel. Dans l'inventivité dont font preuve les personnes, jusqu'au plus grand âge, pour préserver leur statut de donneur, on observe à quel point le fait de donner ne réside pas tant dans ce qu'on donne que dans ce qui affirme l'existence du donneur: je donne, donc j'existe. Et quand les vicissitudes du grand âge érodent l'aptitude à tenir le rôle de donneur, c'est ce sentiment même d'exister qui est ébranlé. A moins que n'intervienne, comme on l'a vu, l'idée d'un échange différé associé au cycle de vie.

Cette lecture appelle cependant quelques questions. Dans quelle mesure est-il vrai, comme le veut la théorie, que le statut de donneur est associé à une position de supériorité dans le système de l'échange? En d'autres termes, ceux qui reçoivent le don initial se conçoivent-ils comme des «obligés »? Reconnaissent-ils ce don comme dette? Par ailleurs, si le don initial est celui de la vie, en quoi consiste le contre-don? Quand intervient-il? Est-il alors, en quelque sorte, automatique?

A observer à quel point préserver ou récupérer un statut de donneur semble un enjeu pour les parents, on en déduirait volontiers qu'il leur confère quelque supériorité. Mais le paradoxe est que, quand on se place dans une perspective synchronique, les enfants ne le vivent pas ainsi: mineurs, ils perçoivent bien l'asymétrie de la relation aux parents, le rapport de force et la contrainte, mais pas la dette; une fois majeurs, et émancipés de la contrainte, ils ne sont pas dupes du jeu des parents: tout en appréciant l'aide que ceux-ci leur apportent, ils savent pertinemment que leurs parents tirent un large profit de leur don, en particulier parce que ce don leur assure une place dans la famille. Le plan imaginaire - le don fait exister le donneur en lui assurant une appartenance et en attestant sa compétence - n'est pas pleinement homologue au plan relationnel - le donneur assure sa présence dans le système familial mais le don ne tire pas créance.

Et pourtant le sentiment de dette existe, qui se manifeste clairement vers le milieu de la vie, dans la génération dite pivot en ce qu'elle a des parents âgés et des enfants qui peu à peu s'émancipent. Plusieurs études montrent qu'à cette étape de la vie, un certain consensus s'établit sous la forme du paradoxe formé par les deux convictions suivantes: d'une part: nos enfants ne nous doivent rien; de l'autre: nous avons une dette envers nos vieux parents ${ }^{14}$. Ce qu'ils ont donné et donnent encore à leurs enfants se mesure selon eux à l'aune de la gratuité; ce qu'ils ont reçu de leurs parents resurgit comme dette alors que ceux-ci entrent dans le grand âge. Et c'est bien alors que s'observe quelque chose qui ressemble à une mobilisation en vue d'apporter le contre-don: il y a d'abord les signes précurseurs d'une attention aiguisée, d'une inquiétude:

${ }^{14}$ Voir par exemple la belle étude de Josette Coenen-Hutter, Jean Kellerhals et Malik von Allmen, Les réseaux de solidarité dans la famille, Lausanne, Réalités sociales, 1994. 
«Comment va ta mère?

- Très bien, elle est très indépendante, très active. Mais tu sais, elle a 84 ans, elle devient fragile, alors on s'inquiète toujours un peu.»

Vient alors la mise en place d'un filet de protection; par exemple, les enfants veillent à ne pas tous partir en vacances en même temps, afin que toujours l'un d'eux soit à proximité du vieillard; on installe un système de télé-alarme, on engage des aides à domicile. Nos études ont montré que souvent l'aide apportée, loin de se satisfaire de répondre à une nécessité, anticipe le besoin ou les effets possibles de risques accrus (la chute, l'accident).

Le contre-don s'exprime dans une disponibilité bien plus que dans une prise en charge. Car d'un côté, la norme moderne de l'autonomie garde sa vigueur: la personne âgée se doit de tout faire pour préserver son indépendance mais, de l'autre, l'enfant veut se montrer prêt à intervenir, le cas échéant, lorsque cette autonomie est malmenée.

Pourtant, le sentiment de dette envers leurs parents qu'expriment les personnes dans la cinquantaine ne paraît pas répondre, ou ne plus répondre seulement à un impératif catégorique: «ce sont mes parents, donc ...». Cet absolu moral peut intervenir, mais de manière abâtardie, sous la forme: "c'est quand même (c'est malgré tout) mon père / ma mère », locution pleine du non-dit: «quoi qu'il /elle ait fait, quoi qu'il se soit passé, c'est quand même celui/celle qui a donné la vie». Ce n'est pas tant, semble-t-il, le don de la vie qui fonde le sentiment de dette, que la qualité de la relation qui s'est développée à partir du moment où la vie est créée et se développe. La dette relève aujourd'hui bien moins d'un commandement moral que de la reconnaissance de ce que les parents ont donné de la naissance jusqu'à l'émancipation de l'enfant, reconnaissance au double sens de prise de conscience et de sentiment de gratitude.

Constatons finalement que si la famille a servi de matériau pour l'élaboration des mythes de la création, ces mythes sont loin de rendre compte de la nature du don et de la structure et de la dynamique des échanges dans la relation filiale des familles contemporaines.

\section{Professeur honoraire}

Département de sociologie

Directeur de recherche au Centre Interfacultaire de Gérontologie

Université de Genève

Christian.Lalive@socio.unige.ch 